\title{
p53/PGC-1 $\alpha$-mediated mitochondrial dysfunction promotes PC3 prostate cancer cell apoptosis
}

\author{
JIULING LI ${ }^{1}$, YANY LI ${ }^{1}$, LANLAN CHEN ${ }^{2}$, BINGBING YU ${ }^{1}$, YANAN XUE ${ }^{1}$, \\ RUI GUO ${ }^{1}$, JING SU ${ }^{1}$, YANAN LIU ${ }^{1}$ and LIANKUN SUN ${ }^{1}$ \\ ${ }^{1}$ Department of Pathophysiology, Prostate Diseases Prevention and Treatment Research Center, \\ College of Basic Medical Sciences; ${ }^{2}$ School of Clinical Medicine, Jilin University, Changchun, Jilin 130021, P.R. China
}

Received June 19, 2019; Accepted March 05, 2020

DOI: $10.3892 / \mathrm{mmr} .2020 .11121$

\begin{abstract}
Data for p53 mutation in prostate cancer in The Cancer Genome Atlas database revealed that $>85 \%$ of p53 mutations occurred in the p53 DNA binding domain. These mutations not only severely damage the function of the p53 protein, but also reduce the disease-free survival of patients. Peroxisome proliferator-activated receptor $\gamma$ coactivator- $1 \alpha$ (PGC-1 $\alpha$ ) is involved in the regulation of mitochondrial function and is highly expressed in prostate cancer PC3 and DU145 cells with p53 deletion or mutation. However, whether p53 negatively regulates PGC- $1 \alpha$ in prostate cancer cells remains to be elucidated. In the present study, p53 overexpression was induced in prostate cancer PC3 cells. Subsequently, the expression levels of PGC-1 $\alpha$ and alterations to mitochondrial function were assessed. Moreover, PGC-1 $\alpha$ was activated in prostate cancer PC3 cells using ZLN005 to investigate alterations to mitochondrial function and cell apoptosis. The present study revealed that p53 decreased the expression and nuclear localization of the PGC- $1 \alpha$ protein and induced mitochondrial dysfunction. Activation of PGC- $1 \alpha$ partially reversed p53-mediated mitochondrial dysfunction. Inhibition of the $\mathrm{p} 53 / \mathrm{PGC}-1 \alpha$ pathway on mitochondrial biogenesis and fission-/fusion-associated gene and protein expression were associated with mitochondrial dysfunction. p53/PGC-1 $\alpha$-mediated mitochondrial dysfunction promoted apoptosis of PC3 prostate cancer cells. The results indicated that PGC- $1 \alpha$ is an essential target of p53-induced apoptosis in prostate cancer cells and indicated that targeting PGC- $1 \alpha$ may provide a new therapeutic strategy for prostate cancer.
\end{abstract}

Correspondence to: Professor Liankun Sun or Professor Yanan Liu, Department of Pathophysiology, Prostate Diseases Prevention and Treatment Research Center, College of Basic Medical Sciences, Jilin University, 126 Xinmin Street, Chaoyang, Changchun, Jilin 130021, P.R. China

E-mail:sunlk@jlu.edu.cn

E-mail: ynliu@jlu.edu.cn

Key words: peroxisome proliferator-activated receptor $\gamma$ coactivator-1 $\alpha$, p53, mitochondria, ZLN005, prostate cancer, apoptosis

\section{Introduction}

Prostate cancer is the most common malignant tumor in men, with a mortality rate second to lung cancer (1). Nearly 1.3 million new prostate cancer cases and 359,000 associated deaths were reported worldwide in 2018 (2). Although surgery or radiotherapy can effectively improve the prognosis and prolong the survival of prostate cancer patients, the recurrence and metastasis rates remain high. Therefore, identification of novel potential anticancer targets for the development of therapeutic treatments is critical.

p53 is a classic tumor suppressor. Overexpression of p53 not only inhibits the growth of prostate cancer cells but also enhances the chemosensitivity of prostate cancer cells $(3,4)$. Several studies have demonstrated that peroxisome proliferator-activated receptor $\gamma$ coactivator- $1 \alpha$ (PGC-1 $\alpha)$ is expressed at high levels in p53-mutant or p53-deleted tumor cells and have revealed that PGC-1 $\alpha$ plays a role in cancer progression. For example, Shiota et al (5) reported that PGC-1 $\alpha$ was highly expressed in prostate cancer PC3 and DU145 cells with p53 deletion or mutation and promoted tumor cell growth. Ogasawara et al (6) revealed that PGC-1 $\alpha$ was highly expressed in p53-deficient chronic lymphocytic leukemia cells and maintained normal mitochondrial function. The R72 variant of mutant p53 promoted tumor metabolism and metastasis by enhancing the function of PGC-1 $\alpha$ (7). These findings indicate that the deletion or mutation of p53 may contribute to the enhancement of PGC- $1 \alpha$ expression and function in tumor cells. However, the effect of wild-type p53 on PGC-1 $\alpha$ in tumor cells is unclear.

PGC-1 $1 \alpha$ is a member of the peroxisome proliferatoractivated receptor $\gamma$ coactivator 1 family that coordinates the activity of transcription factors to modulate energy metabolism and other cellular processes (8). As a primary regulator of mitochondrial biogenesis, PGC- $1 \alpha$ functions in the activation of the nuclear respiratory factor 1 (NRF1) transcription factor, leading to the transcription of both nuclear-encoded mitochondrial genes (such as $S D H A$ ) and the transcription factor A, mitochondrial (TFAM) $(9,10)$. PGC-1 $\alpha$ is also involved in the regulation of mitochondrial fission/fusion; PGC-1 $\alpha$ promotes mitochondrial fusion through transcriptional activation of mitofusin $(M F N) 1$ and 2 and affects mitochondrial fission via direct regulation of dynamin-related 
protein 1 (DRP1) expression by binding to its promoter (11-14). Peng et al (15) revealed that rotenone reduces TFAM, MFN2 and DRP1 expression by inhibiting PGC-1 $\alpha$ in pheochromocytoma PC12 cells, resulting in decreased mitochondrial copy number, imbalance of fission/fusion and cell death. Thus, PGC-1 $\alpha$-mediated regulation of mitochondrial biogenesis and fission/fusion not only affects mitochondrial function but also determines the survival and death of tumor cells. These findings indicate that PGC- $1 \alpha$ may be a potential target for cancer therapy.

The present study examined the effect of p53 on PGC- $1 \alpha$ and revealed that p53 decreased the expression of mitochondrial biogenesis and fission/fusion-associated genes by inhibiting PGC-1 $\alpha$, leading to mitochondrial dysfunction and ultimately apoptosis. These findings revealed that PGC- $1 \alpha$ is a crucial target of p53-induced apoptosis in $\mathrm{PC} 3$ prostate cancer cells and indicated that targeting PGC- $1 \alpha$ may provide a new therapeutic strategy for prostate cancer.

\section{Materials and methods}

Cell culture and reagents. Human prostate cancer cell lines PC3 and DU145 were purchased from the American Type Culture Collection and cultured in RPMI-1640 medium (Gibco; Thermo Fisher Scientific, Inc.) containing 10\% fetal bovine serum (Invitrogen; Thermo Fisher Scientific, Inc.). ZLN005, a transcriptional activator of PGC-1 $\alpha$, was purchased from MedChemExpress LLC. Hoechst 33342 and 3-(4,5-dimethylthiazol-2-yl)-2,5-diphenyltetrazolium bromide (MTT) were purchased from Sigma-Aldrich (Merck KGaA). Anti- $\beta$-actin (cat. no. sc-47778), anti-p53 (cat. no. sc-6243), anti-Mfn1 (cat. no. sc-166644), anti-Mfn2 (cat. no. sc-100560), anti-DRP1 (cat. no. sc-271583), anti-Bak (cat. no. sc-832) and anti-Bcl-2 (cat. no. sc-509) antibodies were purchased from Santa Cruz Biotechnology, Inc. Anti-NRF1 (cat. no. A5547) and anti-TFAM (cat. no. A1926) antibodies were purchased from ABclonal Biotech Co.,Ltd. Anti-PGC-1 $\alpha$ (cat.no.66369-Ig) and anti-SDHA (cat. no. 14865-1-AP) antibodies were purchased from ProteinTech Group, Inc. Anti-cleaved-caspase-3 (product no. 9664) was purchased from Cell Signaling Technology, Inc.

Transfection and drug treatment. The pcDNA3.1 vector (negative control) and the full-length p53 expression vector were purchased from Shanghai GeneChem Co., Ltd. Transfections were performed using TurboFect Transfection Reagent (Thermo Fisher Scientific, Inc.), according to the manufacturer's protocol. Briefly, PC3 cells were seeded $\left(5 \times 10^{5}\right.$ cells/well) into 6-well plates and cultured overnight at $37^{\circ} \mathrm{C}$. Subsequently, cells were transfected with p53 expression vector $(4 \mu \mathrm{g})$ or pcDNA3.1 $(4 \mu \mathrm{g})$. Following transfection for 4-6 $\mathrm{h}$ at $37^{\circ} \mathrm{C}$, the PGC-1 $\alpha$ activator ZLN005 was added and the cells were cultured for $24 \mathrm{~h}$ at $37^{\circ} \mathrm{C}$ prior to subsequent experiments. shRNA sequences targeting human PGC- $1 \alpha$ and a non-target sequence were constructed by GeneChem Co., Ltd. (Shanghai, China.), as previously described (16). The sequences of PGC-1 $\alpha$ shRNA and the non-target shRNA were 5'-GTTATACCTGTGATGCTTT-3' and 5'-TTCTCCGAA CGTGTCACGT-3', respectively. Cells were transfected with shRNAs $(4 \mu \mathrm{g})$ using the GV248 vector (GeneChem, Inc.), according to the aforementioned protocol.
Immunofluorescence staining and confocal laser microscopy. Coverslips were placed in 24-well plates and $8 \times 10^{4}$ cells were added into each well. The transfection efficiency of the p53 plasmid and the co-localization of p53 with PGC-1 $\alpha$ were examined using indirect immunofluorescence, as previously described (17). Stained cells were observed in five random fields of view using an FV1000 confocal laser microscope (Olympus Corporation; magnification, x200).

Reverse transcription-quantitative $(R T-q) P C R$. Cells were cultured in 6 -well plates at a density of $5 \times 10^{5}$ cells/well. Total RNA was extracted from cells using TRIzol ${ }^{\circledR}$ (Invitrogen; Thermo Fisher Scientific, Inc.), according to the manufacturer's protocol. Subsequently, total RNA was reverse transcribed into cDNA using $0.5 \mu \mathrm{g}$ total RNA and the SuperScript Pre-amplifcation system (Promega Corporation), according to the manufacturer's protocol. qPCR was performed using the CFX96 Touch $^{\mathrm{TM}}$ Real-Time PCR detection system (Bio-Rad Laboratories, Inc.), as previously described (15), and the TransStart Top Green qPCR SuperMix (cat. no. AQ131; Beijing Transgen Biotech Co., Ltd.), with a reaction system consisting of $1 \mu \mathrm{l}$ cDNA, $0.2 \mu \mathrm{M}$ forward primer, $0.2 \mu \mathrm{M}$ reverse primer, $10 \mu \mathrm{l}$ qPCR SuperMix and $8.6 \mu 1$ nuclease-free water. The following thermocycling conditions were used for qPCR: Initial denaturation at $94^{\circ} \mathrm{C}$ for $30 \mathrm{sec}, 40$ cycles of $94^{\circ} \mathrm{C}$ for $5 \mathrm{sec}$ and a final extension at $60^{\circ} \mathrm{C}$ for $30 \mathrm{sec}$. The following primer pairs were used for qPCR: NRF1 forward, 5'-GCTACTTACACCGAGCAT AGTA-3' and reverse, 5'-CTCAAATACATGAGGCCG TTTC-3'; TFAM forward, 5'-TTCCAAGAAGCTAAGGGT GATT-3' and reverse, 5'-AGAAGATCCTTTCGTCCAACT T-3'; SDHA forward, 5'-GTCCCTCCAATTAAACCAAAC G-3' and reverse, 5'-GTTCCGATGTTCTTATGCTTCC-3'; MFN1 forward, 5'-GTGGCAAACAAAGTTTCATGT G-3' and reverse, 5'-CACTAAGGCGTTTACTTCATCG-3'; MFN2 forward, 5'-GTGCTTCTCCCTCAACTATGAC-3' and reverse, 5'-ATCCGAGAGAGAAATGGAACTC-3'; DRP1 forward, 5'-GAGATGGTGTTCAAGAACCAAC-3' and reverse, 5'-CAATAACCTCACAATCTCGCTG-3'; and $\beta$-actin forward, 5'-CCTGGCACCCAGCACAAT-3' and reverse, 5'-GGGCCGGACTCGTCATAC-3'. mRNA expression levels were quantified using the $2^{-\Delta \Delta \mathrm{Cq}}$ method (18) and normalized to the internal reference gene $\beta$-actin.

Western blot analysis. Cells were lysed using RIPA lysis buffer (Takara Bio, Inc.), sonicated at $20 \mathrm{kHz}$ for $30 \mathrm{sec}$ on ice and incubated on ice for $45 \mathrm{~min}$. Cell lysates were centrifuged at $3,000 \mathrm{x} \mathrm{g}$ for $15 \mathrm{~min}$ at $4^{\circ} \mathrm{C}$. Total protein was quantified using the Coomassie G250 assay (Beyotime Institute of Biotechnology). Protein samples (20-30 $\mu \mathrm{g})$ were separated by $12 \% \mathrm{w} / \mathrm{v}$ SDS-PAGE and transferred onto PVDF membranes. The PVDF membranes were blocked with $5 \%$ skim milk powder for $2 \mathrm{~h}$ at room temperature. Subsequently, the membranes were incubated overnight at $4{ }^{\circ} \mathrm{C}$ with primary antibodies (primary antibodies purchased from Santa Cruz Biotechnology, Inc. were diluted at 1:200; primary antibodies purchased from ProteinTech Group, Inc. or Cell Signaling Technology, Inc. were diluted at 1:1,000.). Anti- $\beta$-actin was use as loading control. Following primary incubation, the membranes were incubated with horseradish-peroxidase-conjugated secondary 
antibodies (1:2,000; cat.no. SA00001-1 and SA00001-2; ProteinTech Group, Inc.) for $2 \mathrm{~h}$ at room temperature. Protein bands were visualized using Pierce ECL Western Blotting Substrate (Thermo Fisher Scientific, Inc.) and imaged using a Syngene BioImaging system (Synoptics). Protein expression was quantified using ImageJ software (version 1.48; National Institutes of Health) with $\beta$-actin as the loading control.

Oxygen consumption rate (OCR). OCR was measured using the MitoXpress ${ }^{\circledR}$ Xtra-Oxygen Consumption assay (Luxcel Biosciences Ltd.) as previously reported (19).

Mitochondrial mass. Coverslips were placed in 24-well plates and $8 \times 10^{4}$ cells were added into each well. Mitochondria were stained in live cells using MitoTracker ${ }^{\mathrm{TM}}$ Red CMXRos (Invitrogen; Thermo Fisher Scientific, Inc.), as previously described (16).

Cell viability assays. Cells were cultured $\left(1.2 \times 10^{4}\right.$ cells/well $)$ in 96-well plates overnight at $37^{\circ} \mathrm{C}$ and subsequently cultured for $24 \mathrm{~h}$ at $37^{\circ} \mathrm{C}$ after the addition of various concentrations of ZLN005 (5,10,15 or $20 \mu \mathrm{M})$. MTT assays were performed as previously described (19). Briefly, $10 \mu 1$ MTT $(10 \mathrm{mg} / \mathrm{ml})$ reagent was added to each well and incubated for 4-6 h. The formazan crystals were dissolved with $150 \mu \mathrm{l}$ DMSO and the absorbance was measured at a wavelength of $490 \mathrm{~nm}$.

Annexin V and cell death assay. Cells were cultured in 6-well plates $\left(5 \times 10^{5}\right.$ cells/well) and transfection was performed within $24 \mathrm{~h}$. At 4-6 h post-transfection, ZLN005 $(15 \mu \mathrm{M})$ was added to the culture. After incubation for $24 \mathrm{~h}$ at $37^{\circ} \mathrm{C}$, the Annexin V Apoptosis Detection Kit II (BD Biosciences) was used to measure cellular apoptosis, according to the manufacturer's protocol. Apoptotic cells were detected by flow cytometry using a BD Accuri C6 cytometer (BD Biosciences), and data analysis was conducted using BD Accuri C6 software (version 1.0.264.21; BD Biosciences).

Dataset analysis. All dataset analysis results were obtained from cBioPortal (http://www.cbioportal.org/), which hosts multiple cancer genomics studies, including all of the data from The Cancer Genome Atlas (TCGA). Patient survival was determined by Kaplan-Meier and log-rank analyses and correlation analysis was determined by Spearman and Pearson methods.

Statistical analysis. Each experiment was repeated three times. Results are expressed as the mean \pm standard deviation. One-way analysis of variance was performed to compare results among the groups, followed by Bonferroni's multiple comparison test to determine statistical significance. All statistical analyses were performed using SPSS 24 statistical software (IBM Corp.).

\section{Results}

p53 mutation is associated with decreased disease-free survival in prostate cancer patients. The TCGA data demonstrated that $12 \%$ of prostate cancer patients harbor p53 mutations (Fig. 1A). Most of these mutations occur in the p53 DNA-binding domain and severely impair the function of the p53 protein (Fig. 1B). Survival analysis demonstrated no significant difference in overall survival between patients with p53 mutations and p53 non-mutation patients, but the disease-free survival rate of patients with p53 mutations was decreased compared to p53 non-mutation patients (Fig. 1C). Unlike p53, the mutation rate of PGC- $1 \alpha$ in prostate cancer patients was only $0.8 \%$ and these mutations had a weak effect on the function of PGC-1 $\alpha$ (Fig. S1A); therefore, the finding that PGC-1 $\alpha$ mutation reduced the survival rate requires verification using a larger sample size (Fig. S1B).

p53 inhibits the protein expression and nuclear localization of PGC-1 $\alpha$. The deletion or mutation of p53 may contribute to the enhancement of PGC-1 $\alpha$ expression and function. However, whether p53 negatively regulates PGC- $1 \alpha$ is unclear. To explore the effect of wild-type p53 on PGC-1 $\alpha$, p53-deleted PC3 prostate cancer cells were transfected with a construct that overexpresses p53. Immunofluorescence staining (Fig. 2A) and western blot analysis (Fig. 2B) confirmed that p53 was successfully overexpressed in PC 3 cells transfected with the p53 overexpression vector, while no p53 was detected in negative control (NC) cells. Next, the protein expression and nuclear localization of PGC- $1 \alpha$ in response to p53 overexpression was examined. As revealed in Fig. $2 \mathrm{C}$, a decrease in PGC-1 $\alpha$ protein expression was detected in p53-overexpressing cells compared with the NC cells. Furthermore, immunofluorescence assay demonstrated that the nuclear staining of PGC- $1 \alpha$ observed in NC cells was decreased upon p53 overexpression (Fig. 2D). These results indicated that p53 inhibited the protein expression and nuclear localization of PGC-1 $\alpha$. In addition, correlation analysis demonstrated that p53 and PGC-1 $\alpha$ tended to be negatively associated, which is consistent with the results of the present study, although the P-value was not significant (Fig. S1C).

Inhibition of PGC-1 $\alpha$ is involved in p53-induced mitochondrial dysfunction. Lack of PGC-1 $\alpha$ has been reported to be a major cause of mitochondrial dysfunction (20). OCR and mitochondrial mass were examined to determine the effect of p53 on mitochondrial function. The effect of p53 on OCR in PC3 cells was examined and there was a decrease in OCR by $52 \%$ in PC3 cells transfected with the $\mathrm{p} 53$ overexpression vector compared with NC cells (Fig. 3A). To evaluate the effect of p53 on mitochondrial mass, PC 3 cells were stained with MitoTracker Red, a fluorescence dye that stains mitochondria in a mass-dependent fashion. A marked decrease in mitochondrial mass in p53-overexpressing PC3 cells compared with NC cells was observed (Fig. 3B). These results confirmed that p53 induced mitochondrial dysfunction in PC3 cells.

To examine whether p53-induced mitochondrial dysfunction involves inhibition of PGC- $1 \alpha$, the mitochondrial function in PC 3 cells after activation of PGC-1 $\alpha$ by ZLN005 was examined. First, PC 3 cells were treated with various concentrations of ZLN005. MTT assays indicated that treatment of PC 3 cells with ZLN005 at concentrations of $\leq 15 \mu \mathrm{M}$ resulted in increased cell survival (Fig. 3C). Next, PGC-1 $\alpha$ expression was examined by western blotting. The results indicated that the levels of the PGC- $1 \alpha$ protein were significantly increased as the concentration of ZLN005 increased (Fig. 3D and E). A concentration of $15 \mu \mathrm{M}$, which demonstrated beneficial effects on cell survival, 
A Samples per patient

TP53

Genetic alteration

Missense mutation (unknown significance)

\section{Truncating mutation | No alterations}

(putative driver)

Samples per patient

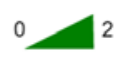

B TP53

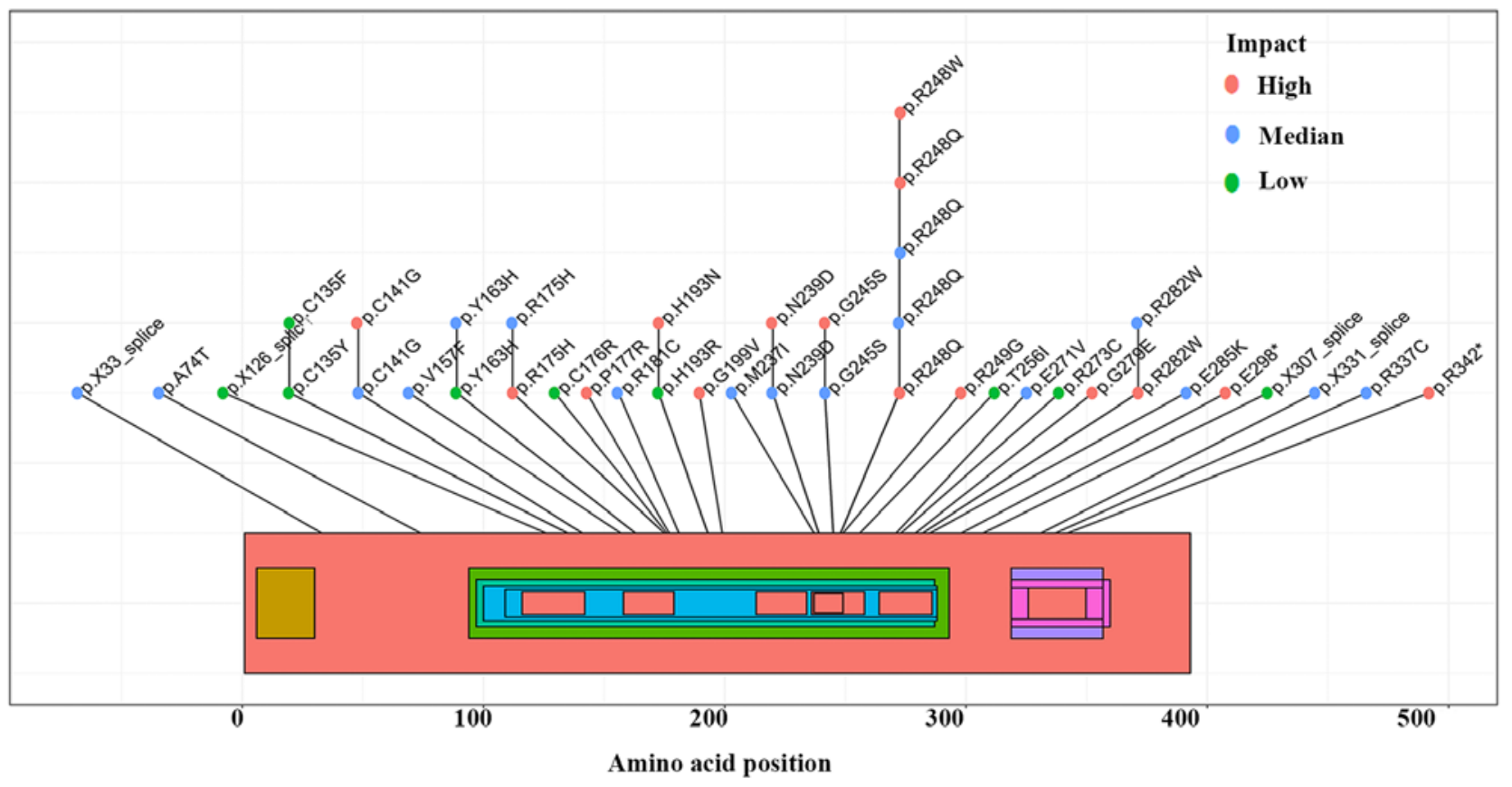

Domain

p53 tumour suppressor family

p53 transactivation domain

p53/RUNT-type transcription factor,DNA-bingding domain superfamily

p53,DNA-binding domain

p53,tetramerisation domain

p53-like transcription factor,DNA-bingding

C

Overall survival kaplan-meier estimate

Disease/progression-free kaplan-meier estimate

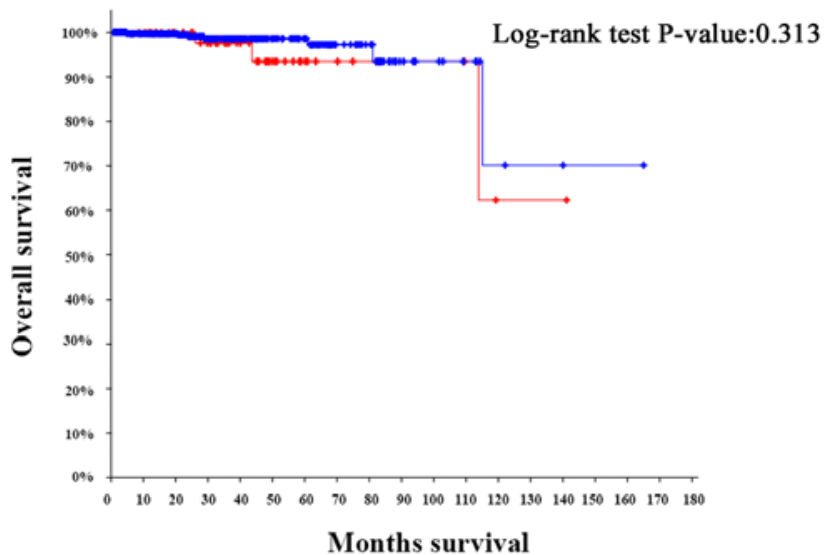

- Case with mutation in TP53

- Case without mutation in TP53

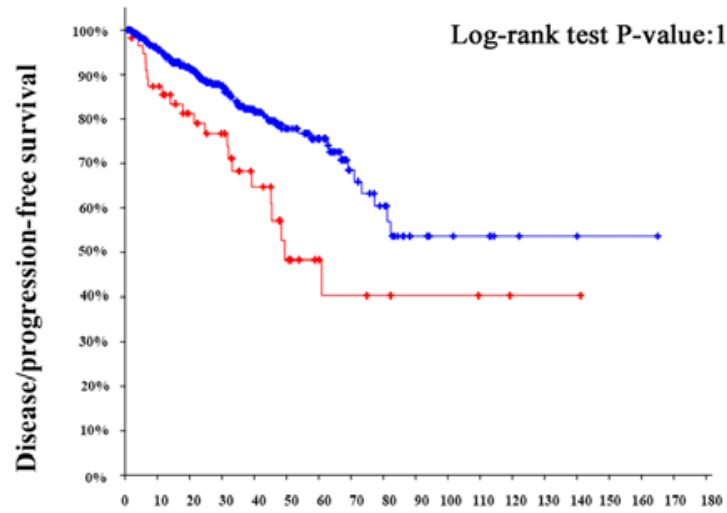

Months survival

- Case with mutation in TP53

- Case without mutation in TP53

Figure 1. p53 mutation is associated with reduced disease-free survival in prostate cancer patients. All datasets can be obtained from cBioPortal (http://www. cbioportal.org/), which hosts multiple cancer genomics studies, including all of the data from The Cancer Genome Atlas. (A) Mutation frequency of p53 in patients with prostate cancer. (B) p53 mutation site and effects on p53 function. (C) Survival analysis of prostate cancer patients with and without p53 mutation. 


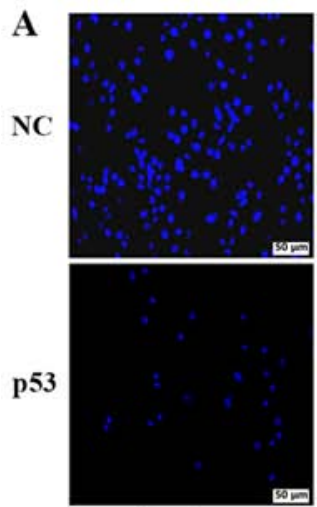

Hochest
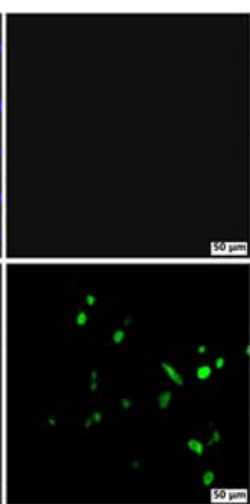

p53

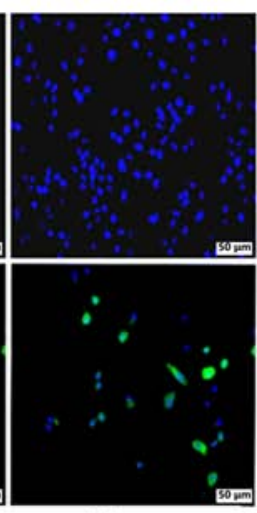

Merge
C

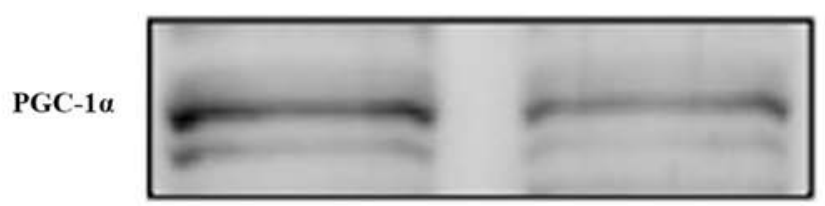

Actin

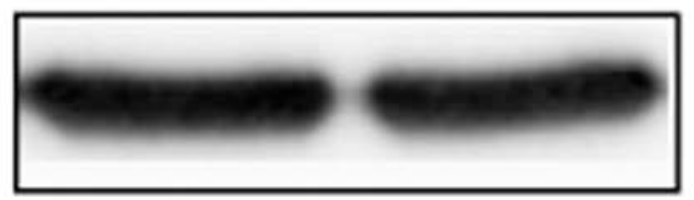

NC

p53
B

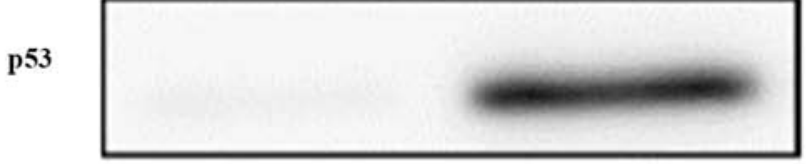

Actin

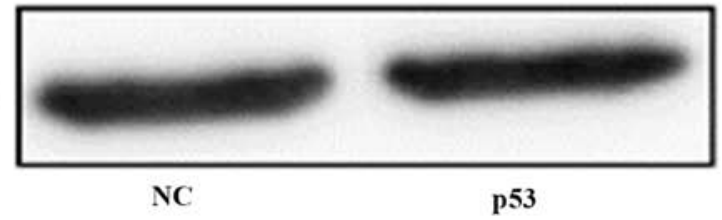

D

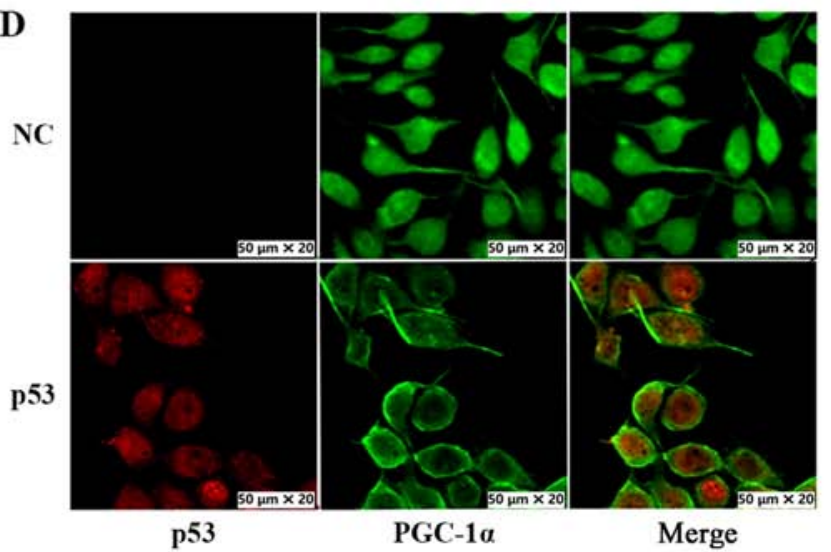

Figure 2. p53 inhibits protein expression and nuclear localization of PGC-1 $\alpha$. PC3 cells were transfected with a p53 overexpression construct for 24 h. (A) Immunofluorescence staining and (B) western blot analysis were used to analyze the efficiency of p53 overexpression. The expression and nuclear localization of PGC-1 $\alpha$ were analyzed by (C) western blot analysis and (D) immunofluorescence staining. NC, negative control; PGC-1 $\alpha$, peroxisome proliferator-activated receptor $\gamma$ coactivator- $1 \alpha$.

was selected for subsequent experiments. p53-overexpressing cells were treated with ZLN005 and the effects on OCR and mitochondrial mass were examined. The OCR was increased by $30 \%$ in PC3 cells with p53 overexpression treated with ZLN005 compared with PC3 cells overexpressing p53 alone (Fig. 3F). Similarly, the mitochondrial mass of PC3 cells with p53 overexpression treated with ZLN005 was also increased compared to cells with p53 overexpression alone (Fig. 3G). These results indicated that inhibition of PGC- $1 \alpha$ may be involved in $\mathrm{p} 53$-induced mitochondrial dysfunction.

p53 decreases the expression of genes and proteins associated to mitochondrial biogenesis and fission/fusion by inhibiting $P G C-1 \alpha$. The results of the present study confirmed that activation of PGC- $1 \alpha$ by ZLN005, a PGC-1 $\alpha$ activator, ameliorated the mitochondrial dysfunction induced by $\mathrm{p} 53$. Since PGC- $1 \alpha$ regulates mitochondrial biogenesis and fission/fusion, the gene expression levels of NRF1, TFAM, SDHA, MFN1/2 and DRP1 were next examined. The results demonstrated that p53 inhibited the expression of NRF1, TFAM, SDHA, MFN1/2 and DRP1 genes, while ZLN005 partially reversed the inhibitory effect of p53 on these genes (Fig. 4A and B). The expression levels of the corresponding proteins were also examined by western blotting. As revealed in Fig. 4C-F, p53 inhibited the expression of NRF1, TFAM, SDHA, Mfn1/2 and DRP1, while ZLN005 partially reversed the inhibitory effect of p53 on the expression of these proteins. These results indicated that p53 decreased the expression of genes and proteins associated to mitochondrial biogenesis and fission/fusion by inhibiting PGC-1 $\alpha$.

p53/PGC-1 $\alpha$-mediated mitochondrial dysfunction promotes apoptosis of PC3 prostate cancer cells. Peng et al (15) revealed that changes in mitochondrial biogenesis and fission/fusion not only affect mitochondrial function but also determine cell survival and death. To investigate the effect of the p53/PGC-1 $\alpha$ pathway on PC3 prostate cancer cells, the rate of apoptosis of $\mathrm{PC} 3$ prostate cancer cells was examined. Flow cytometric analysis revealed that the rate of apoptosis was decreased in p53-overexpressing cells treated with ZLN005 compared with cells only expressing p53 (Fig. 5A and B). Western blot analysis demonstrated that the expression of cleaved caspase- 3 and Bak were slightly decreased in p53-overexpressing cells treated with ZLN005 compared with p53 overexpression alone (Fig. 5C and D). These results indicated that the $\mathrm{p} 53 / \mathrm{PGC}-1 \alpha$ pathway promoted apoptosis and the apoptosis induced by the p53/PGC-1 $\alpha$ pathway may be associated with mitochondrial 
$\mathbf{A}$
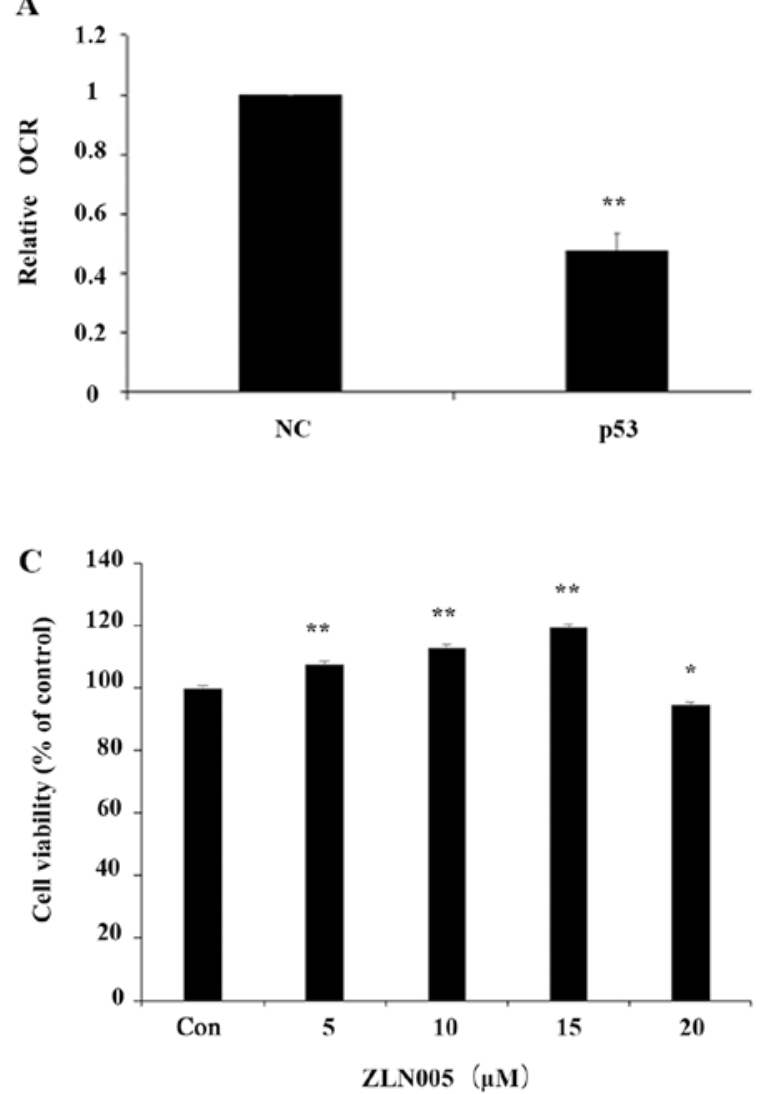

B

NC

p53

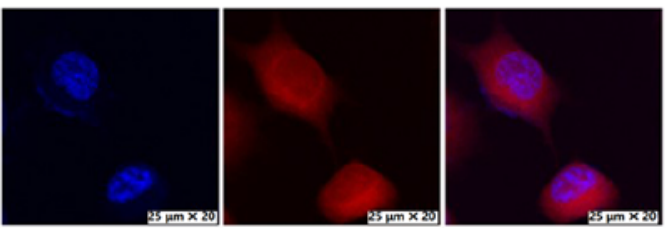

D

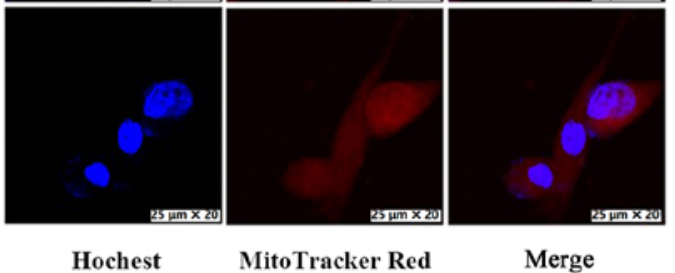

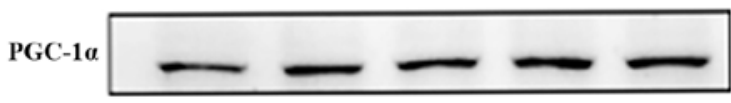

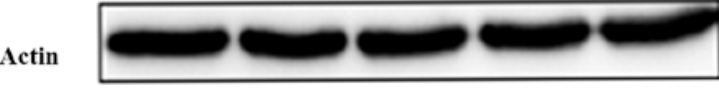

$\begin{array}{lllll}\text { Con } & 5 & 10 & 15 & 20\end{array}$

ZLN005 ( $\mu \mathrm{M})$

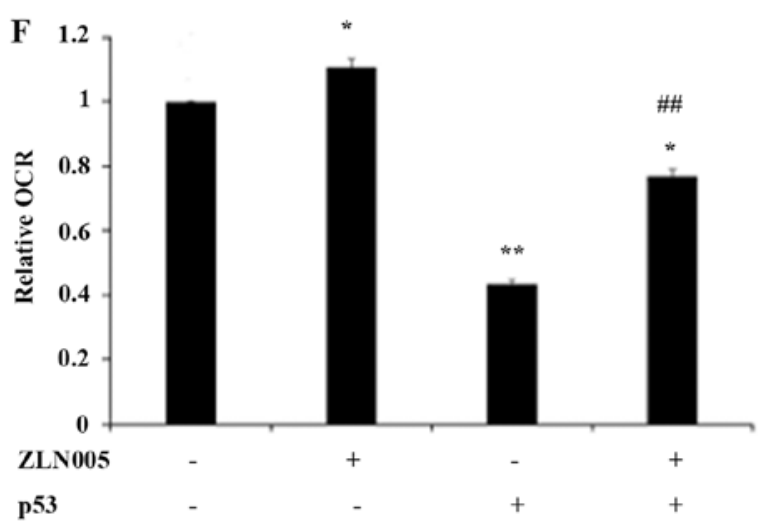

G

Hochest

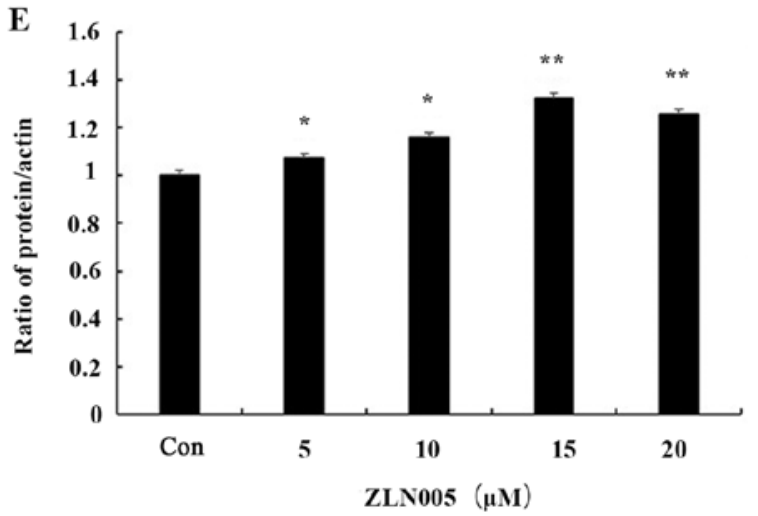

p53

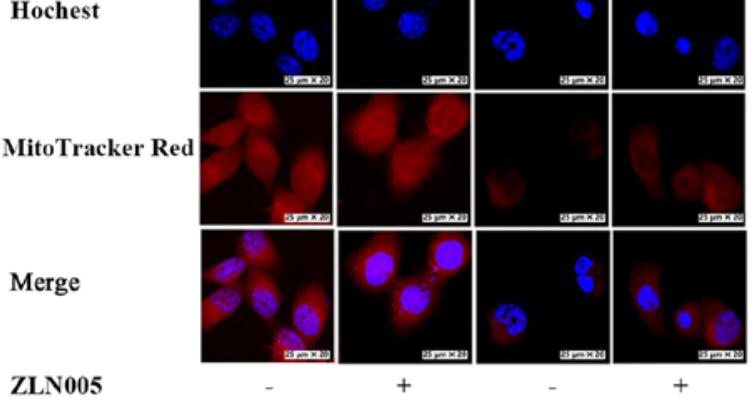

ZLN005

$+$

p53

Figure 3. Inhibition of PGC-1 $\alpha$ is involved in p53-induced mitochondrial dysfunction. (A) Detection of OCR. Data are presented as the mean \pm standard deviation ( $n=3$ ). ${ }^{* *} \mathrm{P}<0.01$ vs. the NC. (B) MitoTracker Red staining for detection of mitochondrial mass. (C) PC3 cells were treated with different concentrations of ZLN005 and cell viability was determined by MTT assay. Data are presented as the mean \pm standard deviation $(\mathrm{n}=3)$. ${ }^{*} \mathrm{P}<0.05$ and ${ }^{* *} \mathrm{P}<0.01$ vs. the control. (D and E) PGC-1 $\alpha$ expression was analyzed by western blotting in PC3 cells treated with ZLN005 (5, 10, 15, or $20 \mu \mathrm{M})$ for $24 \mathrm{~h}$. (F) PC3 cells were transfected with a p53 overexpression construct and treated with/without ZLN005 $(15 \mu \mathrm{M}) 4-6 \mathrm{~h}$ after transfection. Detection of OCR. Data are presented as the mean \pm standard deviation $(\mathrm{n}=3)$. ${ }^{*} \mathrm{P}<0.05$ and ${ }^{* *} \mathrm{P}<0.01$ vs. the control, ${ }^{\# \#} \mathrm{P}<0.01$ vs. p53. (G) MitoTracker Red staining for detection of mitochondrial mass. PGC-1 $\alpha$, peroxisome proliferator-activated receptor $\gamma$ coactivator-1 $\alpha$; OCR, oxygen consumption rates; NC, negative control; Con, control. 


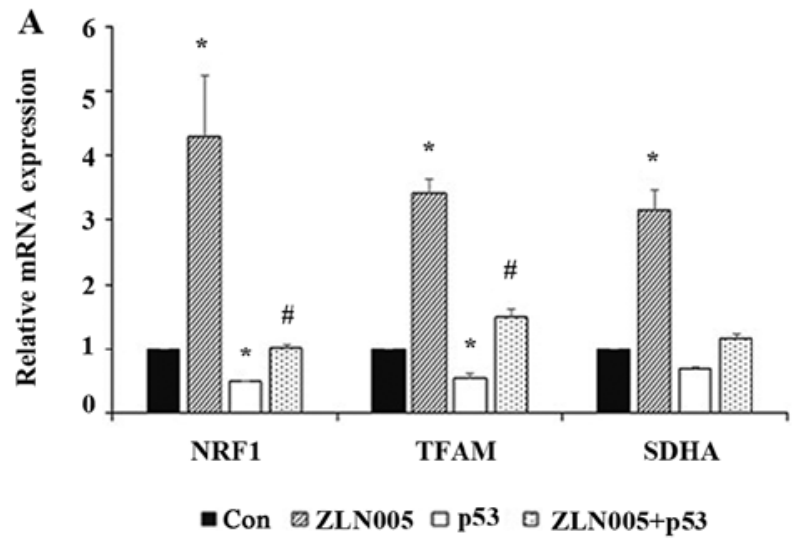

C
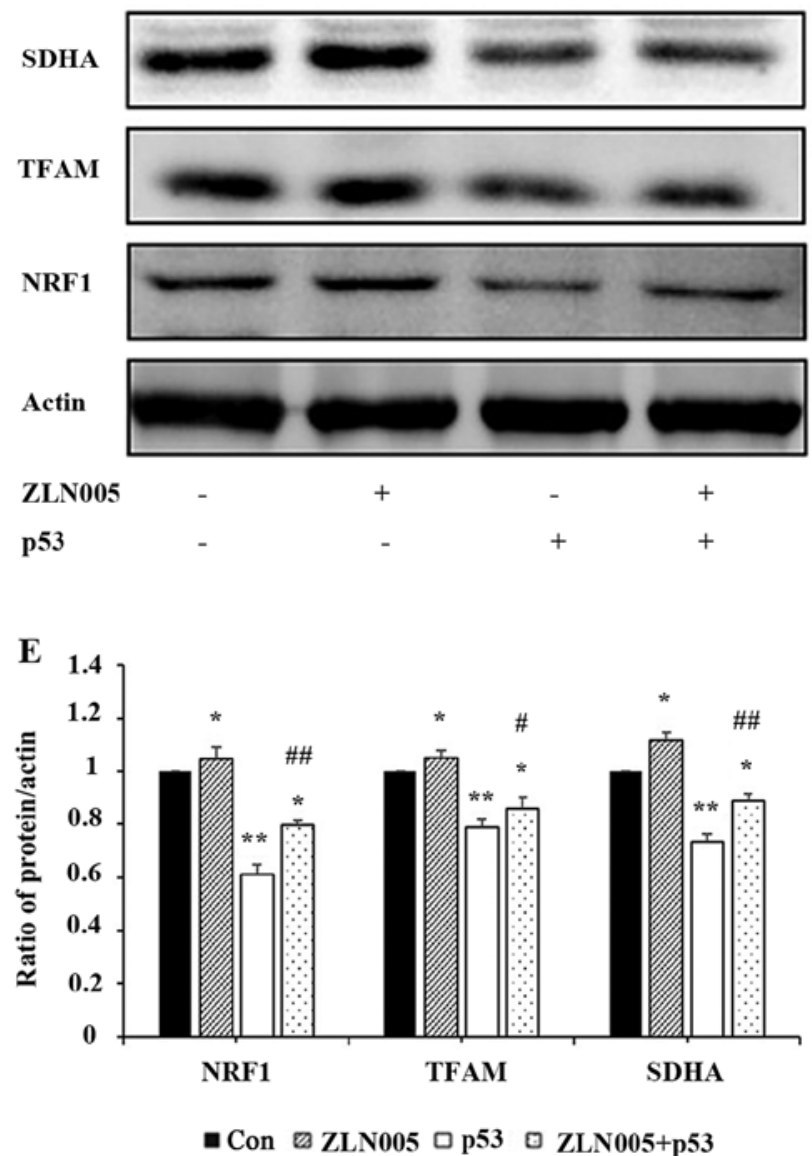

B

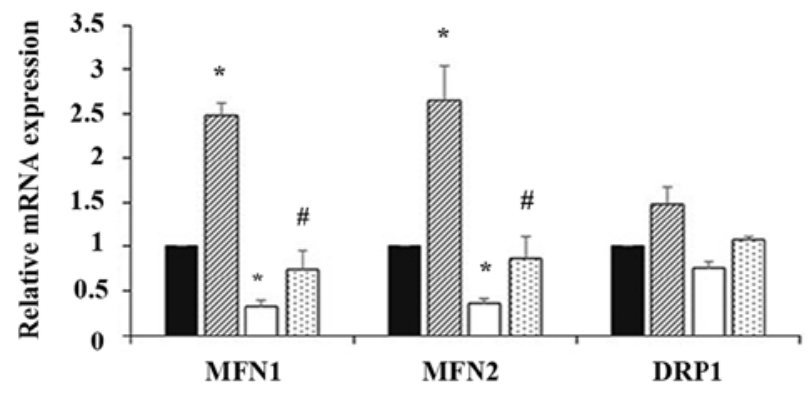

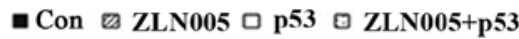

D
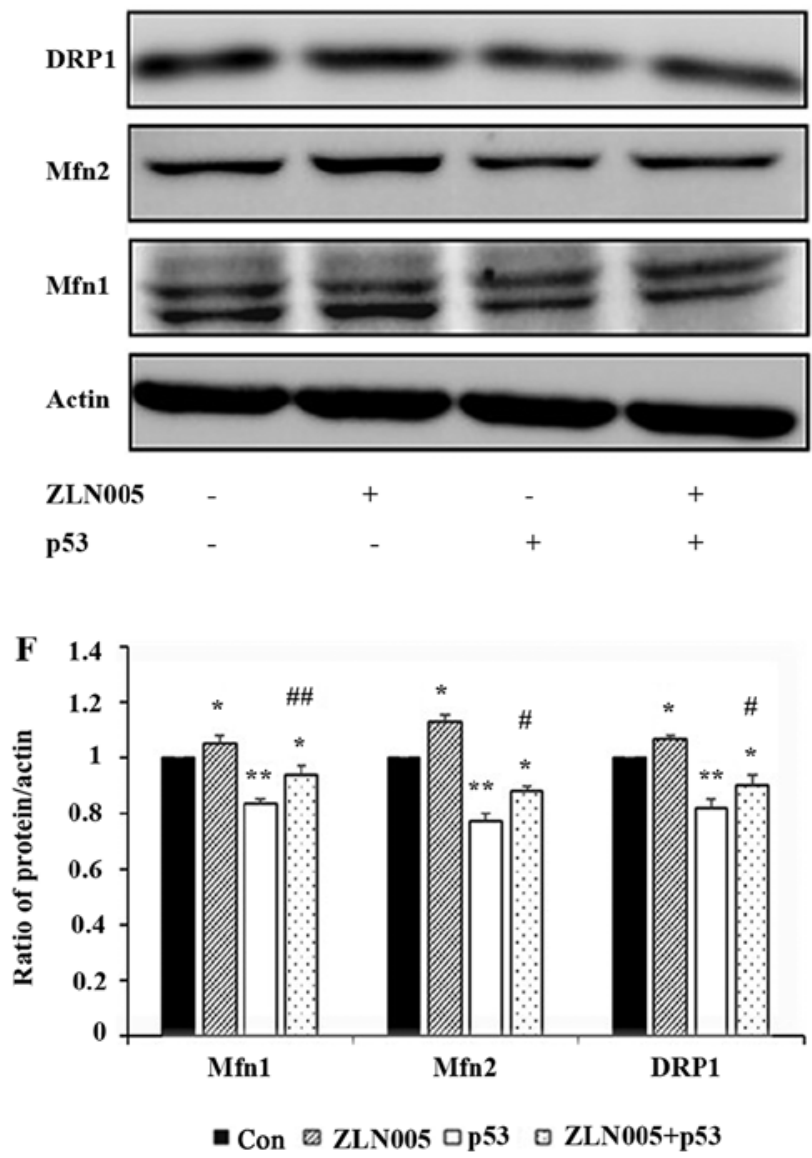

Figure 4. p53 reduces the expression of genes and proteins associated to mitochondrial biogenesis and fission/fusion by inhibiting PGC-1 $\alpha$. The expression levels of (A) mitochondrial biogenesis and (B) fission/fusion genes were detected by RT-qPCR. Data are presented as the mean \pm standard deviation ( $\mathrm{n}=3$ ). ${ }^{*} \mathrm{P}<0.05$ vs. the control; ${ }^{*} \mathrm{P}<0.05$ vs. p53. The expression levels of proteins involved in (C) mitochondrial biogenesis and (D) fission/fusion were analyzed by western blotting. Quantitation of (E) NRF1, TFAM and SDHA, and (F) Mfn1, Mfn2 and DRP1 were assessed using ImageJ software. Data are presented as the mean \pm standard deviation $(\mathrm{n}=3)$. ${ }^{*} \mathrm{P}<0.05$ and ${ }^{* *} \mathrm{P}<0.01$ vs. the control; ${ }^{\#} \mathrm{P}<0.05$ vs. p53, ${ }^{\# \#} \mathrm{P}<0.01$ vs. p53. PGC-1 $\alpha$, peroxisome proliferator-activated receptor $\gamma$ coactivator-1 $\alpha$; RT-qPCR, reverse transcription-quantitative PCR; Con, control; NRF1, nuclear respiratory factor 1; TFAM, transcription factor A, mitochondrial; SDHA, succinate dehydrogenase complex flavoprotein subunit A; Mfn, mitofusin; DRP1, dynamin-related protein 1.

dysfunction. These findings identified PGC-1 $\alpha$ as an essential target of p53-induced apoptosis in prostate cancer cells, indicating that targeting PGC-1 $\alpha$ may serve as a new therapeutic strategy for prostate cancer. Consistent with this possibility, western blot analysis demonstrated that knockout of PGC-1 $\alpha$ promoted the expression of cleaved caspase-3 in PC3 and DU145 prostate cancer cells and decreased the expression of Bcl-2 (Fig. S1D).

\section{Discussion}

The p53 mutation data in prostate cancer from TCGA demonstrated that more than $85 \%$ of p53 mutations occur in the p53 DNA binding domain. These mutations not only severely damage the function of the p53 protein, but also reduced the disease-free survival of prostate cancer patients. The present study explored the functional association between p53 and 
A
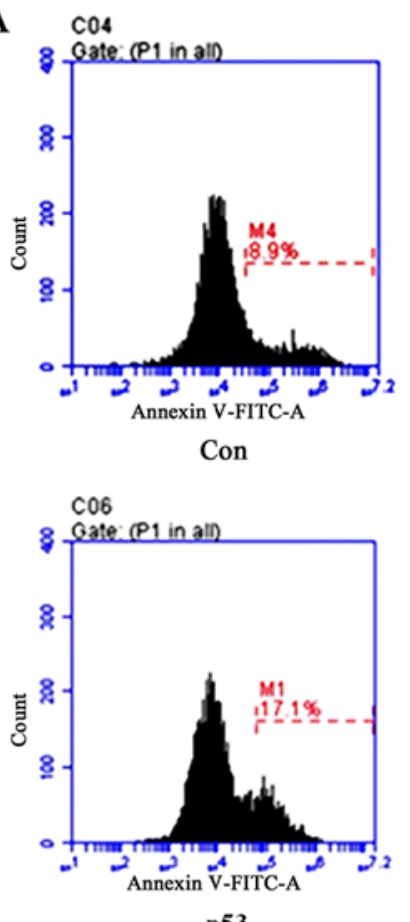

p53

C
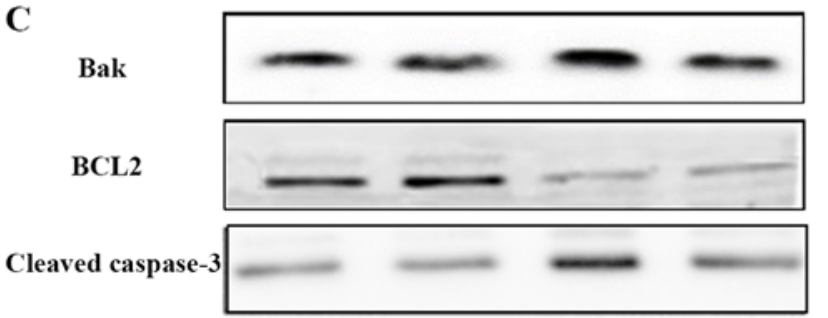

Actin

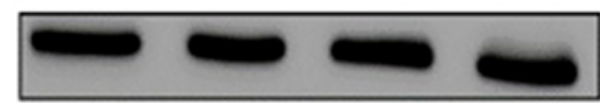

ZLN005

p53
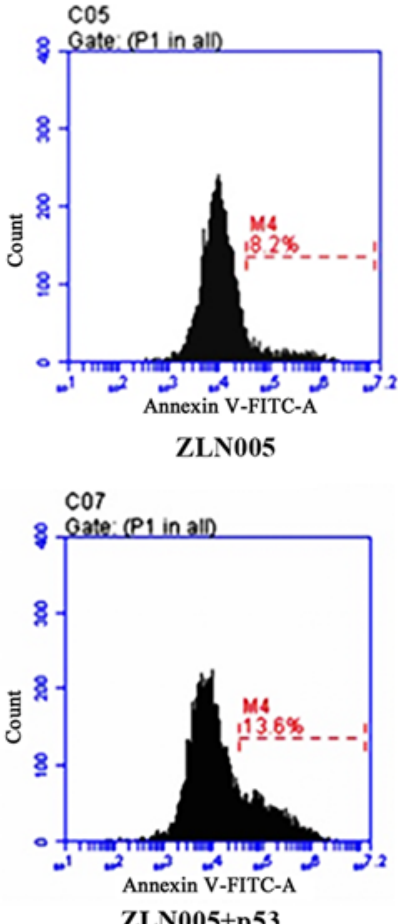

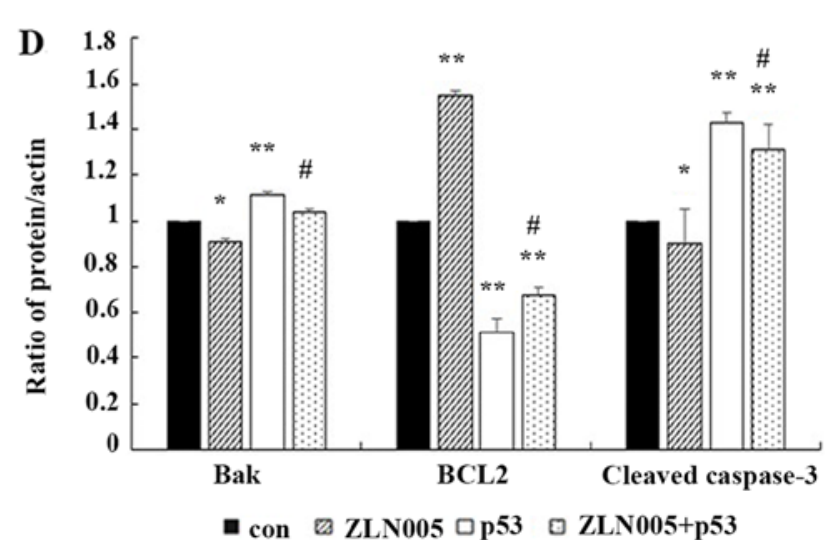

B

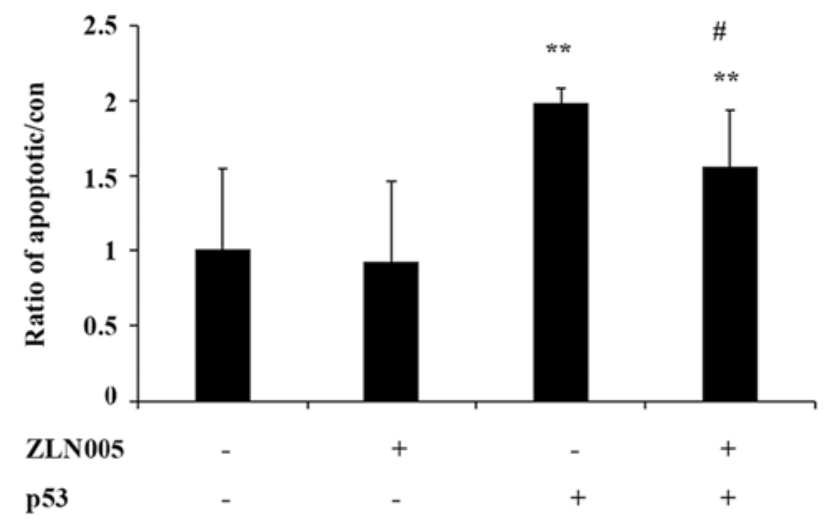<smiles>CCC</smiles>

Figure 5. p53/PGC-1 $\alpha$-mediated mitochondrial dysfunction promotes apoptosis of PC3 prostate cancer cells. (A and B) Detection of apoptosis by Annexin V. (C and D) Cleaved caspase-3, Bak and Bcl-2 were analyzed by western blotting. ${ }^{*} \mathrm{P}<0.05$ and ${ }^{* *} \mathrm{P}<0.01$ vs. the control; ${ }^{*} \mathrm{P}<0.05$ vs. p53. PGC-1 $\alpha$, peroxisome proliferator-activated receptor $\gamma$ coactivator- $1 \alpha$; Con, control.

PGC-1 $\alpha$ by overexpressing p53 in p53-deficient prostate cancer PC3 cells and revealed that p53 inhibited the protein expression and nuclear localization of PGC-1 $\alpha$. The inhibition of PGC-1 $\alpha$ by 553 decreased the expression of genes and proteins associated to mitochondrial biogenesis and fission/fusion and led to mitochondrial dysfunction and apoptosis. These results revealed that PGC- $1 \alpha$ was an essential target of p53-induced apoptosis in prostate cancer cells and indicated that targeting PGC-1 $\alpha$ may provide a new therapeutic strategy for prostate cancer.

A study reported that overexpression of p53 negatively affects the normal mitochondrial homeostasis in HepG 2 cells, but the precise mechanism of mitochondrial dysfunction has not been investigated (21). Another study demonstrated that PGC-1 $\alpha$ promotes prostate cancer cell growth by activating the AR. However, whether the survival and death of prostate cancer cells are determined by mitochondrial function regulated by PGC-1 $\alpha$ remains unclear (5). The present study demonstrated that inhibition of PGC- $1 \alpha$ by p53 induced mitochondrial dysfunction and apoptosis of PC3 cells, indicating an essential involvement of PGC-1 $\alpha$ in p53-mediated control of mitochondrial dysfunction and apoptosis. The results not only revealed that inhibition of PGC- $1 \alpha$ by p53 is associated with mitochondrial dysfunction but also confirmed the importance of PGC-1 $\alpha$ in cell survival.

The present study focused on the regulation of PGC- $1 \alpha$ by p53. Previous studies have demonstrated that p53 transcriptionally inhibits and promotes PGC- $1 \alpha$ in other non-tumor cells $(22,23)$. For example, p53 binds to the repressive-954 and -564 regions of the mouse PPARGC1A promoter and inhibits the expression of PGC-1 $\alpha(22,23)$, However, upon antioxidant glutathione shortage, $\mathrm{p} 53$ is released from the two repressive regions and binds to the -2317 region, which is positively associated to increased PGC-1 $\alpha$ expression (22). The various activities of $\mathrm{p} 53$ on PGC- $1 \alpha$ expression may be associated with redox modification of critical p53 amino acids that affect 
its DNA binding activity (22). The present study revealed that p53 induced a decrease of PGC- $1 \alpha$ protein expression and nuclear localization in PC3 tumor cells. However, Aquilano et al (22) demonstrated that p53 binds to the -1237 region in the human PPARGC1A promoter in SH-SY5Y cells to enhance PGC-1 $\alpha$ expression. Collectively, these results indicate that p53 exhibits various effects on PGC- $1 \alpha$ expression in tumor cells. There are at least two possible reasons for these contradictory activities of p53 on PGC- $1 \alpha$ expression in tumor cells. First, post-transcriptional modification of p53 may result in the binding of p53 to different regions of the PGC-1 $\alpha$ promoter. It was hypothesized that the p53-binding region in the human PPARGC1A promoter may be the functional homolog of the -954 and -564 regions and not the -1237 region in the mouse PPARGC1A promoter. Second, there may be protein-protein interactions between p53 and PGC-1 $\alpha$. Based on the results of the present study, inhibition of PGC-1 $\alpha$ by p53 at the transcriptional level does not fully explain the decreased expression of PGC-1 $\alpha$ in the nucleus. Protein-protein interactions between p53 and PGC-1 $\alpha$ may explain PGC- $1 \alpha$ reduction in the nucleus and this possibility will be the focus of a future study.

The present study identified that activation of PGC- $1 \alpha$ by ZLN005 resulted in amelioration of the mitochondrial dysfunction and apoptosis induced by p53. Activation of PGC-1 $\alpha$ by ZLN005 regulated mitochondrial biogenesis and fission/fusion, which is probably involved in the maintenance of mitochondrial function and promotion of cell growth and survival. Although previous studies have demonstrated the effects of ZLN005 in increasing the expression of PGC-1 $\alpha$, further experiments to evaluate the effect of PGC-1 $\alpha$ overexpression are required, because ZLN005 also transcriptionally promotes genes encoding the deacetylase SIRT1 and antioxidant enzymes SOD1 and HO-1 (24-26). Whether activation of these genes is involved in improving mitochondrial function and apoptosis is unclear.

In conclusion, the findings of the present study demonstrated that p53 decreased the expression of mitochondrial biogenesis and fission-/fusion-associated genes by inhibiting PGC-1 $\alpha$, leading to mitochondrial dysfunction and ultimately apoptosis. The results revealed a pro-cancer effect from PGC- $1 \alpha$ and indicated that PGC- $1 \alpha$ may be a new therapeutic target for PC3 prostate cancer cells. However, the precise regulatory mechanism linking p53 and PGC-1 $\alpha$ remains to be elucidated and requires further investigation.

\section{Acknowledgements}

Not applicable.

\section{Funding}

The present study was supported by the National Natural Science Foundation of China (grant nos. 81772794, 81501982, 81672948 and 81572927), Jilin Provincial Research Foundation for the Development of Science and Technology Projects (grant nos. 20170623021TC and 20160414005GH), Jilin Provincial Industrial Innovation Project (grant no. 2018C052-7) and Jilin University Bethune Plan B Projects (grant nos. 2015222 and 2018A02), Jilin Province Health and Health Technology
Innovation Project (grant no. 2018J061) and the Fundamental Research Funds for the Jilin Universities.

\section{Availability of data and materials}

The datasets used and/or analyzed in the present study are available from the corresponding author on reasonable request.

\section{Authors' contributions}

LS, YL, JS, JL and YNL designed the study. JL and LC collected and analyzed the data. JL, YX, RG and BY performed the experiments. JL drafted the manuscript. YNL, JS and YL reviewed the manuscript. All authors reviewed the manuscript and approved the final manuscript.

\section{Ethics approval and consent to participate}

Not applicable.

\section{Patient consent for publication}

Not applicable.

\section{Competing interests}

The authors declare that they have no competing interests.

\section{References}

1. Siegel RL, Miller KD and Jemal A: Cancer statistics, 2020. CA Cancer J Clin 70: 7-30, 2020.

2. Bray F, Ferlay J, Soerjomataram I, Siegel RL, Torre LA and Jemal A: Global cancer statistics 2018: GLOBOCAN estimates of incidence and mortality worldwide for 36 cancers in 185 countries. CA Cancer J Clin 68: 394-424, 2018.

3. Ji K, Wang B, Shao YT, Zhang L, Liu YN, Shao C, Li XJ, Li X, $\mathrm{Hu} J \mathrm{D}, \mathrm{Zhao} \mathrm{XJ}$, et al: Synergistic suppression of prostatic cancer cells by coexpression of both murine double minute 2 small interfering RNA and wild-type p53 gene in vitro and in vivo. J Pharmacol Exp Ther 338: 173-183, 2011.

4. Chappell WH, Lehmann BD, Terrian DM, Abrams SL, Steelman LS and McCubrey JA: p53 expression controls prostate cancer sensitivity to chemotherapy and the MDM2 inhibitor Nutlin-3. Cell Cycle 11: 4579-4588, 2012.

5. Shiota M, Yokomizo A, Tada Y, Inokuchi J, Tatsugami K, Kuroiwa K, Uchiumi T, Fujimoto N, Seki N and Naito S: Peroxisome proliferator-activated receptor gamma coactivator-1alpha interacts with the androgen receptor (AR) and promotes prostate cancer cell growth by activating the AR. Mol Endocrinol 24: 114-127, 2010.

6. Ogasawara MA, Liu J, Pelicano H, Hammoudi N, Croce CM, Keating MJ and Huang P: Alterations of mitochondrial biogenesis in chronic lymphocytic leukemia cells with loss of p53. Mitochondrion 31: 33-39, 2016.

7. Basu S, Gnanapradeepan K, Barnoud T, Kung CP, Tavecchio M, Scott J, Watters A, Chen Q, Kossenkov AV and Murphy ME: Mutant p53 controls tumor metabolism and metastasis by regulating PGC-1 $\alpha$. Genes Dev 32: 230-243, 2018.

8. Villena JA: New insights into PGC-1 coactivators: Redefining their role in the regulation of mitochondrial function and beyond. FEBS J 282: 647-672, 2015

9. Piantadosi CA and Suliman HB: Transcriptional regulation of SDHa flavoprotein by nuclear respiratory factor-1 prevents pseudo-hypoxia in aerobic cardiac cells. J Biol Chem 283: 10967-10977, 2008.

10. Li PA, Hou X and Hao S: Mitochondrial biogenesis in neurodegeneration. J Neurosci Res 95: 2025-2029, 2017. 
11. Martin OJ, Lai L, Soundarapandian MM, Leone TC, Zorzano A Keller MP, Attie AD, Muoio DM and Kelly DP: A role for peroxisome proliferator-activated receptor $\gamma$ coactivator-1 in the control of mitochondrial dynamics during postnatal cardiac growth. Circ Res 114: 626-636, 2014

12. Cartoni R, Léger B, Hock MB, Praz M, Crettenand A, Pich S, Ziltener JL, Luthi F, Dériaz O, Zorzano A, et al: Mitofusins $1 / 2$ and ERRalpha expression are increased in human skeletal muscle after physical exercise. J Physiol 567: 349-358, 2005.

13. Guo K, Lu J, Huang Y, Wu M, Zhang L, Yu H, Zhang M, Bao Y, $\mathrm{He}$ JC, Chen $\mathrm{H}$ and Jia W: Protective role of PGC-1 $\alpha$ in diabetic nephropathy is associated with the inhibition of ROS through mitochondrial dynamic remodeling. PLoS One 10: e0125176, 2015.

14. Dabrowska A, Venero JL, Iwasawa R, Hankir MK, Rahman S, Boobis A and Hajji N: PGC-1 $\alpha$ controls mitochondrial biogenesis and dynamics in lead-induced neurotoxicity. Aging (Albany NY) 7: 629-647, 2015.

15. Peng K, Yang L, Wang J, Ye F, Dan G, Zhao Y, Cai Y, Cui Z, Ao L, Liu J, et al: The interaction of mitochondrial biogenesis and fission/fusion mediated by PGC-1 $\alpha$ regulates rotenone-induced dopaminergic neurotoxicity. Mol Neurobiol 54: 3783-3797, 2017.

16. Shen L, Sun B, Sheng J, Yu S, Li Y, Xu H, Su J and Sun L: PGCla promotes cisplatin resistance in human ovarian carcinoma cells through upregulation of mitochondrial biogenesis. Int J Oncol 53: 404-416, 2018.

17. Xu L, Xie Q, Qi L, Wang C, Xu N, Liu W, Yu Y, Li S and $\mathrm{Xu}$ Y: Bcl-2 overexpression reduces cisplatin cytotoxicity by decreasing ER-mitochondrial $\mathrm{Ca} 2+$ signaling in SKOV3 cells. Oncol Rep 39: 985-992, 2018.

18. Livak KJ and Schmittgen TD: Analysis of relative gene expression data using real-time quantitative PCR and the 2(-Delta Delta C(T)) method. Methods 25: 402-408, 2001.

19. Wu Y, Gao WN, Xue YN, Zhang LC, Zhang JJ, Lu SY, Yan XY, Yu HM, Su J and Sun LK: SIRT3 aggravates metformin-induced energy stress and apoptosis in ovarian cancer cells. Exp Cell Res 367: 137-149, 2018.
20. Chen X, Zhong J, Dong D, Liu G and Yang P: Endoplasmic reticulum stress-induced CHOP inhibits PGC- $1 \alpha$ and causes mitochondrial dysfunction in diabetic embryopathy. Toxicol Sci 158: 275-285, 2017.

21. Koczor CA, White RC, Zhao P, Zhu L, Fields E and Lewis W: p53 and mitochondrial DNA: Their role in mitochondrial homeostasis and toxicity of antiretrovirals. Am J Pathol 180: 2276-2283, 2012.

22. Aquilano K, Baldelli S, Pagliei B, Cannata SM, Rotilio G and Ciriolo MR: p53 orchestrates the PGC-1 $\alpha$-mediated antioxidant response upon mild redox and metabolic imbalance. Antioxid Redox Signal 18: 386-399, 2013.

23. Sahin E, Colla S, Liesa M, Moslehi J, Müller FL, Guo M, Cooper M, Kotton D, Fabian AJ, Walkey C, et al: Telomere dysfunction induces metabolic and mitochondrial compromise. Nature 470: 359-365, 2011.

24. Zhang LN, Zhou HY, Fu YY, Li YY, Wu F, Gu M, Wu LY, Xia CM, Dong TC, Li JY, et al: Novel small-molecule PGC-1 $\alpha$ transcriptional regulator with beneficial effects on diabetic $\mathrm{db} / \mathrm{db}$ mice. Diabetes 62: 1297-1307, 2013.

25. Li W, Li X, Wang B, Chen Y, Xiao A, Zeng D, Ou D, Yan S, Li W and Zheng Q: ZLN005 protects cardiomyocytes against high glucose-induced cytotoxicity by promoting SIRT1 expression and autophagy. Exp Cell Res 345: 25-36, 2016.

26. Xu Y, Kabba JA, Ruan W, Wang Y, Zhao S, Song X, Zhang L, Li J and Pang T: The PGC-1 $\alpha$ activator ZLN005 ameliorates ischemia-induced neuronal injury in vitro and in vivo. Cell Mol Neurobiol 38: 929-939, 2018.

This work is licensed under a Creative Commons Attribution-NonCommercial-NoDerivatives 4.0 International (CC BY-NC-ND 4.0) License. 